Review

\title{
Theranostic Imaging of the Kinases and Proteases that Modulate Cell Death and Survival
}

\author{
Howard H. Chen ${ }^{1,2}$, Hushan Yuan ${ }^{3}$, Lee Josephson ${ }^{1,3}$, David E. Sosnovik ${ }^{1,2 \varpi}$ \\ 1. Martinos Center for Biomedical Imaging, Massachusetts General Hospital, Harvard Medical School, Boston MA \\ 2. Center for Molecular Imaging Research, Massachusetts General Hospital, Harvard Medical School, Boston MA \\ 3. Center for Translational Nuclear Medicine and Molecular Imaging, Massachusetts General Hospital, Harvard Medical \\ School, Boston MA
}

$\triangle$ Corresponding author: David E. Sosnovik. 149 13th Street, Charlestown MA 02129617 724-3407; sosnovik@nmr.mgh. harvard.edu

(c) Ivyspring International Publisher. This is an open-access article distributed under the terms of the Creative Commons License (http://creativecommons.org/ licenses/by-nc-nd/3.0/). Reproduction is permitted for personal, noncommercial use, provided that the article is in whole, unmodified, and properly cited.

Received: 2012.01.11; Accepted: 2012.01.31; Published: 2012.02.08

\begin{abstract}
Several signaling cascades are involved in cell death, with a significant amount of crosstalk between them. Despite the complexity of these cascades several key pro-survival and pro-death players have been identified. These include PI3-kinase, AKT and caspase-3. Here we review the approaches used to date to perform molecular imaging of these important targets. We focus in particular on approaches that include the possibility of modulating the activity of these kinases and proteases in a theranostic approach.
\end{abstract}

Key words: Molecular Imaging, Theranostic, Cell Death, Apoptosis, PI3K

\section{Introduction}

Several techniques have been developed to image cell death and viability. These include the detection of changes in the pharmacokinetics and distribution of exogenous contrast agents (for example delayed gadolinium enhancement in the myocardium), and the detection of altered metabolic activity in tissue (for example radiolabeled glucose uptake in tumors). More recently, molecular imaging techniques have been developed to image the mechanism of cell death. Several ligands, most notably annexin $\mathrm{V}$ and synaptotagmin, that bind to the surface of apoptotic cells have been used to image apoptosis in vivo by MRI and SPECT [1-5]. In addition, a DNA-binding gadolinium chelate (Gd-TO) has been developed to specifically image necrotic cell death in vivo [6,7]. Imaging the intracellular signaling involved in cell death and survival, however, has proven more challenging. Nevertheless, several novel techniques to image key players in cell death/survival signaling have been developed.

Here we review recent progress in the molecular imaging of three central kinases/proteases involved in cell death and survival: 1) phosphatidylinositol-3-kinase (PI3-kinase or PI3K); 2) the serine/threonine kinase AKT and; 3) the cysteine protease caspase-3. Particular emphasis is placed on the role of these agents in cardiovascular disease and cancer. We use the term "theranostic" fairly broadly to include any construct that can be imaged noninvasively in vivo and can modulate the biological activity of a kinase/protease. In some cases the imaging ligand and the therapeutic moiety may thus be identical, and in other cases the imaging ligand may serve only as a delivery vehicle for a separate therapeutic moiety. The appeal of the latter type of constructs (diagnostic delivery vehicle with attached therapeutic) lies in 
their breadth. A library of theranostic agents can be generated by attaching a variety of therapeutic moieties to the agent. The challenge of these types of theranostic constructs is that they are larger, more complex, and require a stable release mechanism for the therapeutic. As discussed below, initial progress in the field has thus been made largely with the former type of theranostic constructs, where the imaging ligand serves a dual diagnostic and therapeutic purpose.

\section{A Brief Overview of Cell Death/Survival Signaling}

Cells can die via apoptosis, necrosis and autophagy [8]. The signaling pathways involved in all three of these pathways are extremely complex and beyond the scope of this review. Moreover, a significant amount of crosstalk exists between these pathways, producing a complex and interconnected system of signals. Nevertheless, several key nodes in these pathways have been identified including PI3K, AKT and caspase-3 (Figure 1).
The PI3-kinases are a family of lipid kinases that are able to phosphorylate the 3-hydroxyl group of the phosphoinositidyl ring [9]. Three classes of PI3K have been described. Class 1A PI3K is the focus of this paper and plays a major role in cell death and survival, as well as autophagy via mTOR signaling. Class 1A PI3K has a regulatory subunit (p85) and a catalytic subunit (p110). The activation of PI3K is produced by the binding of growth factors such as HER2, IGF and EGRF to their receptor tyrosine kinases. Activation of the receptor tyrosine kinases results in an interaction with the p85 regulatory subunit of PI3K and removes its inhibitory effect on the p110 catalytic subunit. Negative feedback from downstream molecules, and the activity of the tumor suppressor PTEN, keep PI3K activation in balance [10]. Class 2 PI3K are catalytic enzymes that function downstream to activated EGF receptors, play a role in cell death/survival, and have no regulatory/adapter subunit. Class 3 PI3Ks, like class 1 , contain a regulatory $(\mathrm{p} 150)$ and catalytic (Vps34, $100 \mathrm{kDa}$ ) subunit and play an important role in cellular autophagy.

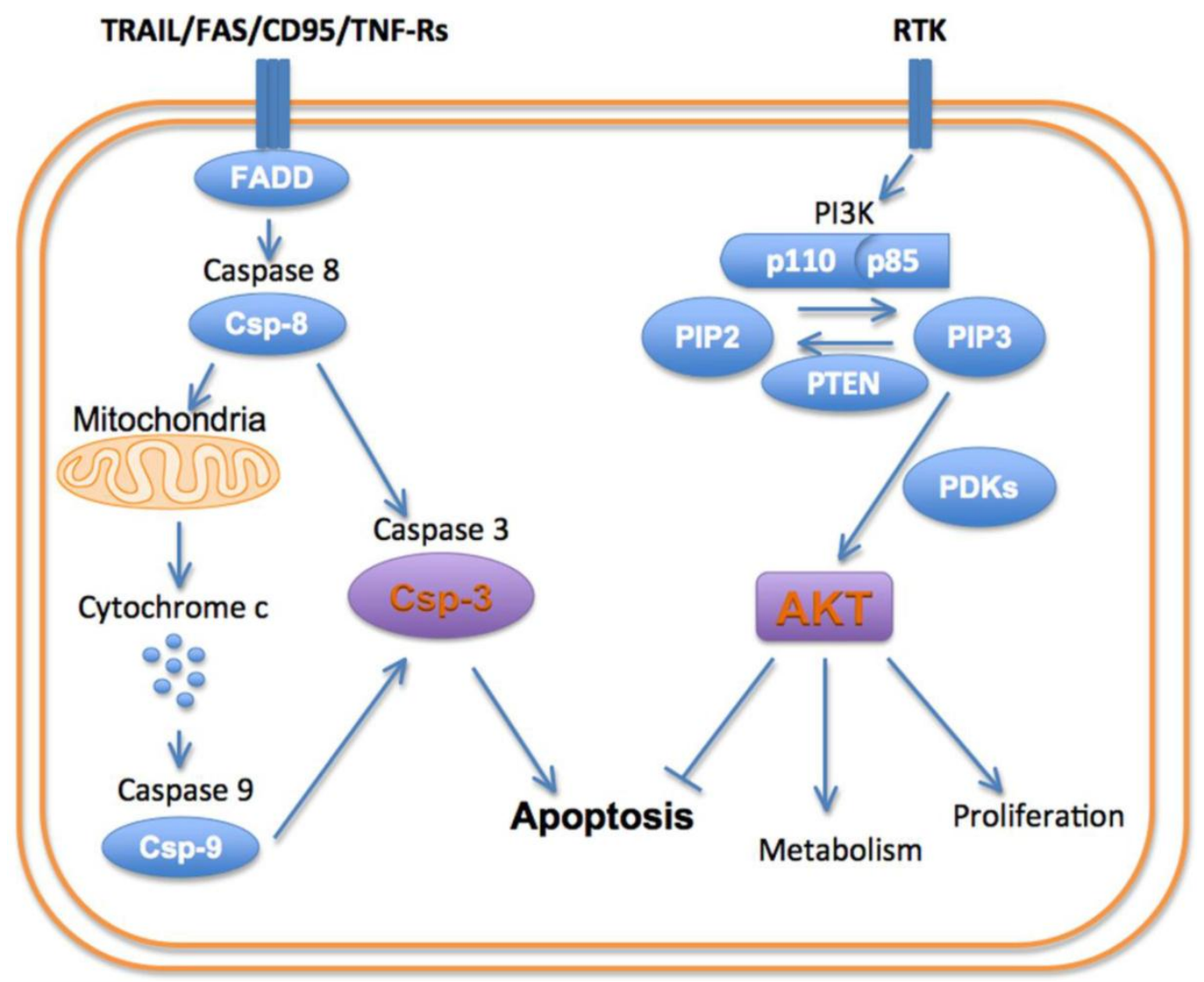

Figure I: Schematic of principal cell death/survival pathways. 
Activation of PI3K produces a robust pro-growth and survival effect, principally by activating the serine/threonine kinase AKT [11]. The activation of PI3K converts phosphatidylinositol-bisphosphate (PIP2) into phosphatidylinositol-triphosphate (PIP3). This in turn recruits AKT and the kinase PDK-1 (phosphoinositidyl dependent kinase 1) to the cell membrane, where AKT is phosphorylated and activated. Activated AKT exerts a myriad of effects on the cell: AKT stimulates protein synthesis and cell growth by activating mTOR (mammalian target of rapamycin). (It should be noted that mTOR plays a major role in autophagy signaling and thus plays a highly multi-faceted role in cell death). AKT promotes cellular proliferation by promoting the cell cycle proteins (c-Myc and cyclin D1) and inactivating the cell cycle inhibitors (p27 and p21). Functioning indirectly through the mTOR complex (mTORC1), AKT also regulates cell proliferation and hypertrophy. AKT inhibits the pro-apoptotic genes (BAD and BIM) thus promoting cell survival [10-12].

The pro-survival role of AKT in the cell is opposed by a family of cysteine-aspartate proteases, or caspases, which play a pivotal role in cell death by apoptosis [8]. Apoptosis can be initiated via an intrinsic or extrinsic pathway, with different initiator caspases involved. The intrinsic pathway typically is initiated by the interaction of cytochrome $\mathrm{c}$ with caspase-9. This pathway plays a major role in cardiomyocyte death in ischemic injury. The extrinsic or death receptor pathway involves caspase- 8 and plays an important role in inflammatory conditions and chemotherapy. Both the intrinsic and extrinsic apoptotic pathways lead to the activation of caspase-3, which is the principal effector or executioner caspase in the cell [8]. In addition to executing the apoptotic program, caspase- 3 is able to cleave sarcomeric proteins in the myocardium, leading to a loss of function independent of cell death [13].

The pivotal role played by PI3K, AKT and caspase-3 in cell death/survival has been shown in transgenic animal models, by using chemical inhibitors and by insights provided from genomic analysis of human cancer. Reduced PTEN expression and mutations in PIK3A (gene on chromosome 3 encoding for the p110 subunit) are found in numerous human cancers [10]. These tumor cells are resistant to apoptosis due to hyperactivity of the PI3K-AKT pathway. The activation of AKT, frequently observed in human tumor cells, can promote growth factor-mediated cell survival directly by inactivating several proapoptotic factors (BAD, procaspase-9 and Forkhead/FKHR transcription factors), and indirectly by activating transcription factors that upregulate anti-apoptotic genes (cyclic-AMP response element-binding protein (CREB), Nuclear Factor-kB).

In the heart, the activation of the PI3K/AKT cascade by IGF-1 has a profound cardioprotective effect in acute ischemia [14]. Conversely, the inhibition of PI3K by wortmannin (Wm) promotes apoptosis and exacerbates cell death in the heart during ischemia [12]. Wm has been extensively studied as a chemotherapeutic agent in vitro, and recent data show that it also exerts a robust anti-inflammatory effect that may prove useful in rheumatological syndromes and other inflammatory conditions [10, 15, 16]. The inhibition of caspase- 3 can be achieved with small molecules and has been shown to be extremely cardioprotective in ischemia and heart failure [17-19]. Transient overexpression of AKT in the myocardium during ischemic injury is also highly protective [20]. Chronic overexpression of AKT, however, leads to excessive negative feedback of PI3K and is harmful [21]. This underscores the complexity of the signaling involved in cell death/survival and the need for novel imaging tools to better understand how to modulate these processes.

\section{Molecular Imaging of PI3-Kinase}

Numerous inhibitors of PI3K have been developed and are being studied in a variety of cancers [10]. Some are pure PI3K inhibitors, while others inhibit both PI3K and mTOR. Conceivably, several of these inhibitors might be suitable ligands for imaging PI3K, but this will need to be established in future studies. The greatest experience to date is with the natural PI3K inhibitor Wortmannin. Wm is a steroid-like viridin metabolite derived from the fungus Penicillium wortmanni. It is a broad and powerful inhibitor of all classes of PI3K with an IC50 in the low nanomolar range. Wm irreversibly inactivates PI3K through a covalent interaction between $\mathrm{C} 20$ on its furan ring and a lysine residue on the ATP binding pocket of the p110 catalytic subunit of PI3K [22]. Unmodified Wm, however, is not a useful ligand for either conventional or theranostic molecular imaging. It is highly lipophilic, reacts extremely rapidly with both the cellular and non-cellular constituents of the blood, and has a very short blood half-life. Modified Wms have thus been developed to image and modulate PI3K activity in cancer and inflammation.

Wm has been modified at both its C11 and C20 positions to produce a library of theranostics. The fluorochrome NBD, has been attached to the C11 position of Wm to yield Wm-NBD [23]. Like Wm, NBD is a small, uncharged and lipophilic material. Wm-NBD thus maintains the properties of Wm, including its low nanomolar IC50 for PI3K. However, 
the presence of NBD on Wm allows the intracellular fate and location of the construct to be imaged with fluorescence and immunohistochemical techniques [23]. The modification of $\mathrm{Wm}$ at its $\mathrm{C} 20$ position inactivates it. However, depending on the nature of the modification, the C20-modified $\mathrm{Wm}$ can reactivate itself $[24,25]$. These Wms are thus self-activating viridins (SAV). The nature of the $\mathrm{C} 20$ modification influences the rate of self-activation. With an N-methyl pyridine at C20, the half-time of Wm formation is approximately 2 hours. With an alkyl group based tertiary amines at C20, the half-time is 8-10 hours [24, 25]. While the IC50 of the alkyl C20 modified Wms is reduced slightly, the prolonged nature of their release produced a stronger antiproliferative effect in vitro than unmodified Wm $[24,25]$.

Modification of Wm at C20 also allows it to be attached to a fluorescently labeled dextran chain. Modification at C20 thus not only regulates self-activation but also produces a soluble Wm construct with the biodistribution properties of the dextran chain. In vivo imaging with this theranostic platform has been performed with a C11 (NBD) and C20 (alkyl, $70 \mathrm{kDa}$ dextran-Cy5.5) modified construct (Figure 2) [25]. Intravital microscopy of the Cy5.5 moiety showed that the agent moved from the vascular to the interstitial compartments with a half-life of 1.3 hours (Figure 2).
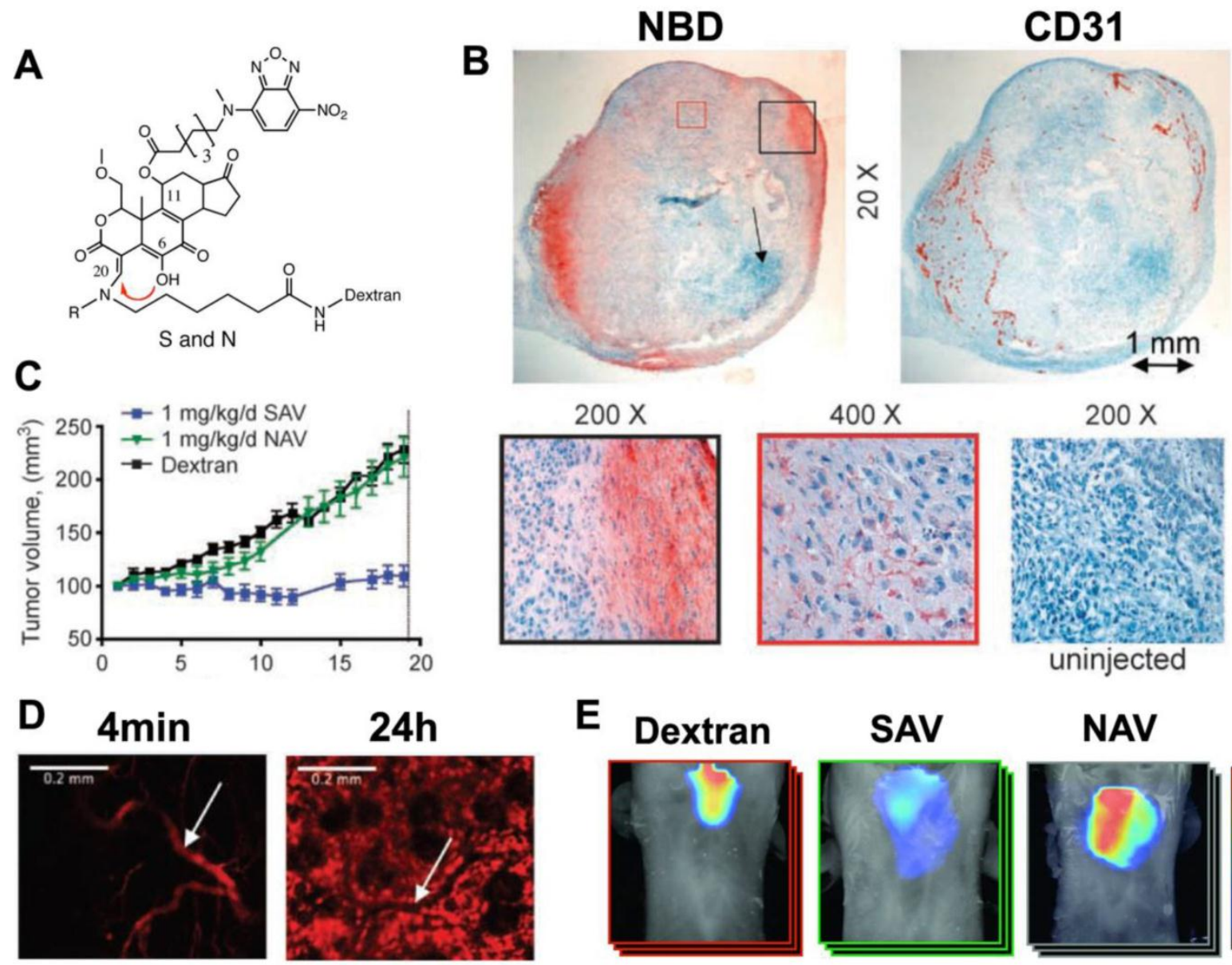

Figure 2: Theranostic imaging of PI3-kinase with a self-activating viridin (SAV). (A) The SAV consists of wortmannin modified at the CII and $\mathrm{C} 20$ positions. The modification at position $\mathrm{CI}$ I resulted in the addition of the fluorochrome, NBD, while position C20 (red arrow) is modified to allow self-activation and conjugation to a dextran chain. When the leaving group (R) at $\mathrm{C}_{2} 0$ is $\mathrm{CH}_{3}$, the self-activating construct (SAV) is formed. When $\mathrm{R}=\mathrm{H}$ the construct is non-activating (NAV). (B) Uptake of injected SAV into the vascularized portions of an implanted xenograft tumor in a mouse [25]. Immunohistochemistry of NBD reveals that the accumulation of wortmannin correlates strongly with CD3I expression. (C) Mice injected with the SAV construct (blue line) showed a complete arrest in tumor growth, which was not seen with NAV or dextran. (D) Intravital microscopy of the Cy5.5 moiety revealed that the kinetics of SAV were dominated by the $70 \mathrm{kDa}$ dextran chain. 4 minutes after injection the probe was exclusively intravascular, but by 24 hours it has accumulated robustly in the interstitial space. (white arrow = blood vessel) [25]. (E) Anti-inflammatory effects of SAV in a mouse asthma model [15]. Whole-body fluorescence tomography images of the thoracic region show differential protease activity in the lungs. Mice injected with SAV show a marked reduction in protease activity in the lungs. Reproduced with permission. 
Mice implanted with an A549 tumor xenograft and injected with $1 \mathrm{mg} / \mathrm{kg} /$ day of the SAV construct showed a complete elimination of tumor growth [25]. This was not seen in mice injected with a non-activating Wm construct (NAV) or with dextran. The NBD moiety on the C11 position of the SAV construct showed that the injected theranostic reached the vascularized portions of the tumor, where the activated Wm-NBD was released and affected tumor growth (Figure 2) [25].

Transgenic knockout mice lacking PI3K show a reduction in neutrophil and macrophage activity. In particular, the oxidative burst in neutrophils is markedly attenuated in these animals. The ability of the SAV Wm construct to modulate immune related diseases has been shown in mouse models of inflammatory arthritis and asthma [15, 16]. Mice injected with $0.75 \mathrm{mg} / \mathrm{kg} /$ day of the construct did not develop arthritis in a serum transfer model, unlike the mice not injected with the agent [16]. Likewise, the administration of the SAV construct significantly reduced pulmonary inflammation in a murine asthma model (Figure 2) [15]. It should be noted that the injected dose of SAV needed for immune modulation is significantly lower than that needed to exert an antiproliferative effect. This raises the possibility of using SAV as an anti-inflammatory agent and minimizing any deleterious effects in healthy tissues.

The optical imaging approaches described above are extremely useful in the preclinical setting but have limited translational potential. Nevertheless, they demonstrate the potential of a PI3K theranostic and lay down a pathway towards clinical translation. Modification of the $\mathrm{C} 11$ position with a radioactive label such as ${ }^{11} \mathrm{C}$ or ${ }^{18} \mathrm{~F}$ would result in a highly translatable PET-detectable probe. (The specific activity of the agent would need to be tailored to the theranostic dose). Other PI3K inhibitors may likewise be suited to PET imaging. Ultimately, a probe that is able to distinguish between the activated and non-activated forms of PI3K could prove most useful. Many routes to theranostic imaging of PI3K thus exist and could play an important role in a large variety of conditions.

\section{Molecular Imaging of AKT}

Molecular imaging of AKT in vivo has been performed in mice using a bioluminescent reporter approach. Bioluminescent AKT reporters (BAR) consist of the AKTpep and FHA2 domains of AKT, flanked on either side by the $\mathrm{N}$-terminal and C-terminal domains of firefly luciferase [26]. Phosphorylation of the AKTpep domain causes it to interact with the FHA2 domain, sterically hindering the formation of a functional luciferase (Figure 3). AKT activation thus decreases the luminescent signal from the construct and can be used to image AKT activation/deactivation in vitro and in mice in vivo (Figure 3) $[26,27]$. More recently a membrane bound version of this split reporter has been described [28]. This is based on the fact that the activation of AKT involves its translocation to the cell membrane. The modified AKT reporter has been reported to be more quantitative and sensitive than the original construct [28].
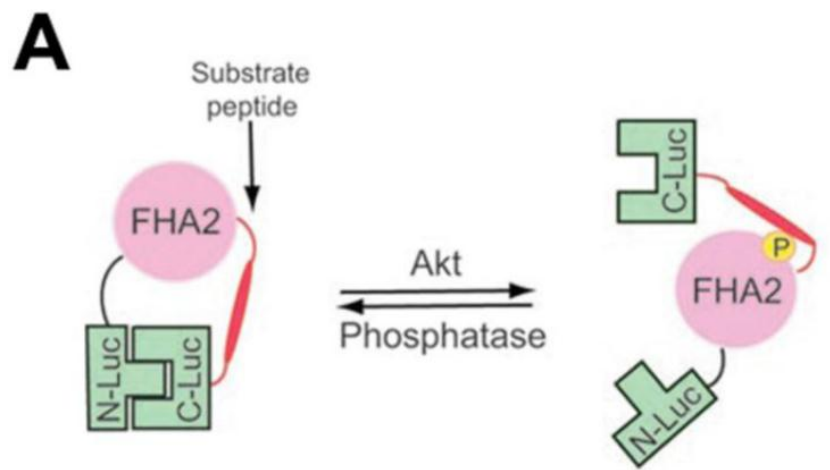

Kinase OFF, Light ON Kinase ON, Light OFF

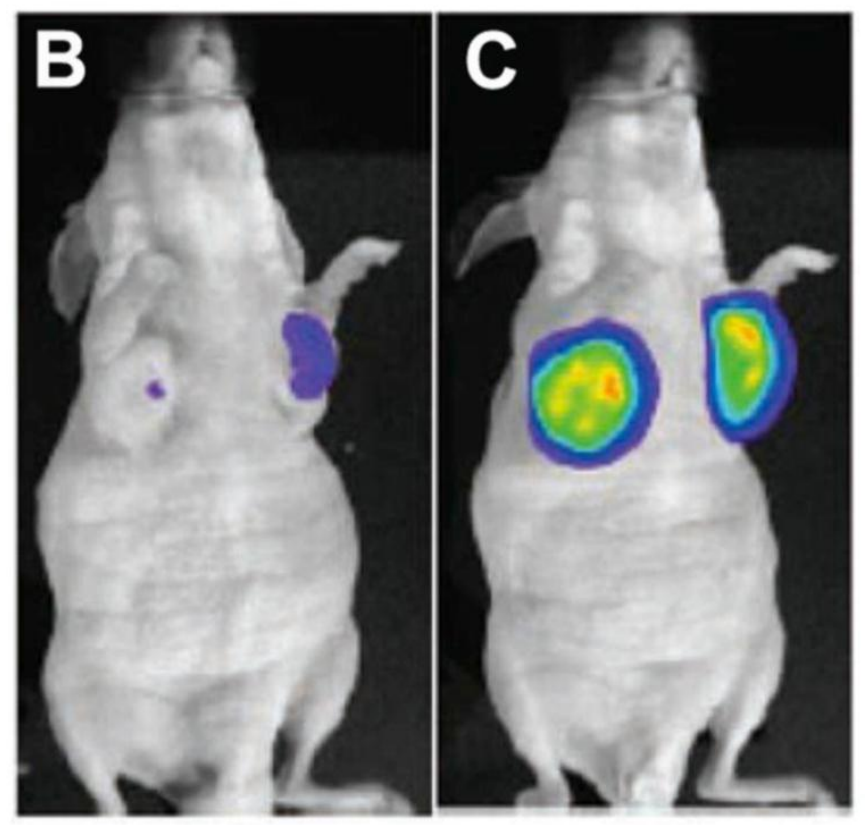

Figure 3: Bioluminescent AKT reporter (BAR). (A) The phosphorylation of AKT leads to a conformational change in the construct and prevents the association of the $N$ and $C$ terminal domains of the reporter [26]. Activation of AKT thus leads to a reduction in the signal. $(B, C)$ Mouse implanted with xenografts transfected with the BAR construct. (B) Little signal is seen before treatment. (C) A large signal is seen in mice treated with the AKT inhibitor perifosine $[26,27]$. Reproduced with permission $[26,27]$. 
A similar approach (conformation dependent reporter complementation) has been used to image a variety of kinases [29-31], as well as proteases such as caspase-3 [32]. While extremely elegant, and of major utility in the preclinical arena, the translational potential of this approach is unclear. Conceivably it might be possible for a clinically-detectable PET reporter to be used to report kinase activity, but this has yet to be shown. Theranostic approaches will also need to be developed. While many challenges remain, the central importance of AKT in cell growth and signaling make the development of theranostic agents to this molecule highly appealing.

\section{Molecular Imaging of Caspase-3}

Caspase- 3 is the key effector protease of the apoptotic cascade. Three approaches have been developed to image caspase-3 including: 1) reporter gene approaches, 2) activatable fluorochromes and, 3) radiolabeled caspase inhibitors [33]. Most of the reporter and activatable constructs developed are based on the cleavage of a peptide linker at the caspase- 3 recognition site DEVD (Asp-Glu-Val-Asp) [34-37]. This leads to conformational changes in the construct such as the dequenching of a flanking pair of fluorochromes/quencher or the generation of a functional reporter [34-37]. To gain access to the cell these agents must incorporate a cell penetrating capability, such as the incorporation of a cell penetrating peptide. This elegant approach has allowed in vivo imaging of caspase- 3 activation to be performed in small animals and in structures such as the retina of the eye (Figure 4) [34-37]. Several limitations of this approach, however, bear mention. The intracellular trafficking of these constructs is difficult to control and accumulation in lysosomes may lead to non-specific activation [33]. Furthermore the other effector caspases (caspases $6,7)$ can also activate these probes to some degree, although less so than caspase-3. The largest limitation of optical based caspase-3 imaging, however, lies in the limited potential for translation. The use of a thymidine kinase PET reporter to image caspase-3 activity in vivo has been attempted but was less sensitive than the use of fluorescent and bioluminescent reporters (Figure 4) [35]. Nevertheless, this raises the possibility of improved and translatable PET reporter approaches being used in the future to image caspase-3 activity.

The use of small chemical inhibitors to directly image caspase-3 provides alternative strategy for clinical translation. The most commonly used ligands are the isatin (1H-indole-2,3-dione) sulfonamide analogs [38-40], which have an IC50 for caspase-3 inhibition in the nanomolar range. Several ${ }^{18}$ F-labeled isatin analogues have been used to image caspase-3 activity in small animal models in vivo (Figure 4).
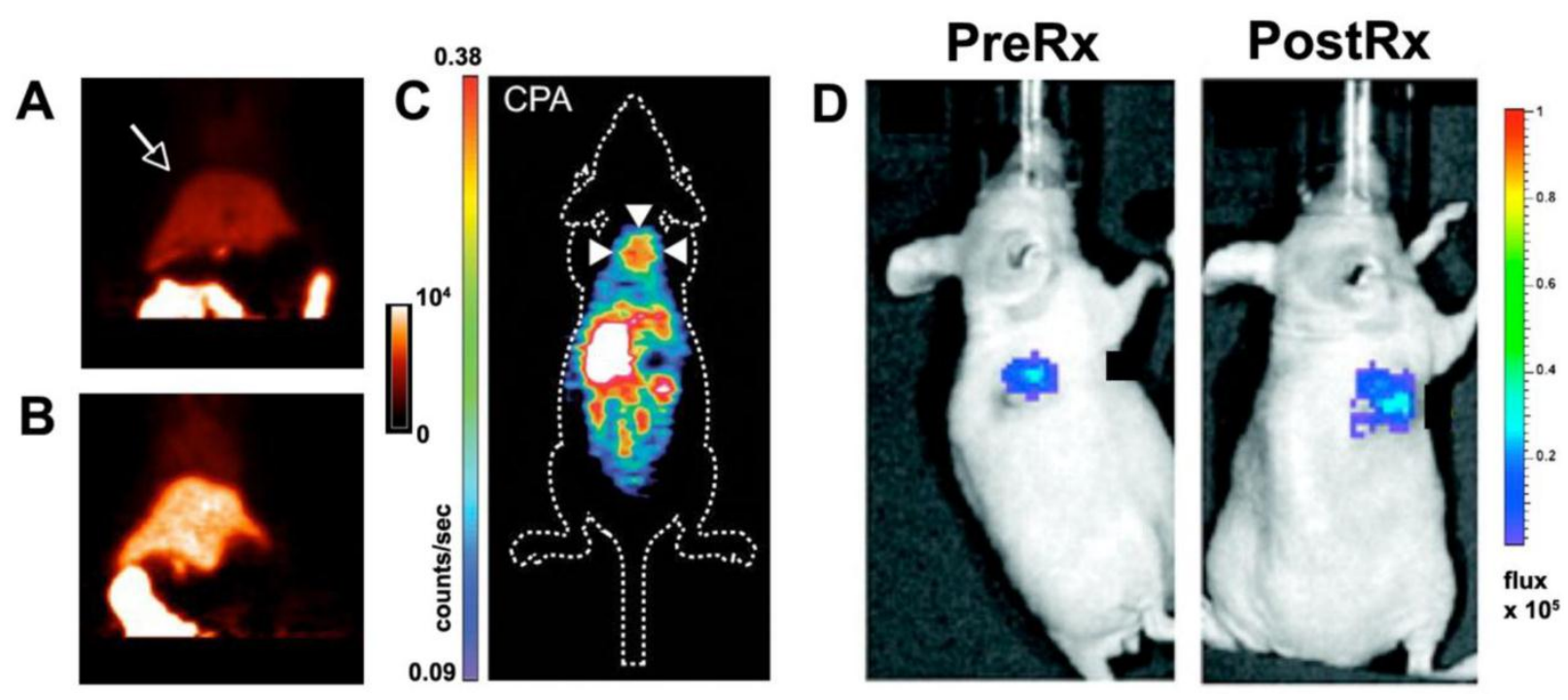

Figure 4: Molecular imaging of caspase-3. (A, B) Rats injected with a $18 \mathrm{~F}$ labeled isatin analog. PET imaging shows that the signal in the liver (arrow) is significantly higher in a rat treated with cycloheximide (B) than in an untreated control animal (A) [38]. (C) Apoptosis in a xenograft tumor (arrows) imaged with PET after chemotherapy and the injection of an $18 \mathrm{~F}$ labeled isatin analog [39]. (D) Luciferase reporter activated by caspase-3 [35]. A xenograft tumor transfected with the reporter emits a low signal before chemotherapy and a robust signal after treatment. Reproduced with permission [35, 38, 39]. 
Uptake of the agent was increased in a rat model of cycloheximide induced liver injury [38]. Likewise, uptake of the agent in tumor xenografts was two-fold higher 24 hours after chemotherapy than in control animals [39]. While extremely promising, these studies have raised some important issues. Firstly, a large amount of background uptake of the isatin probes is seen in the liver and other abdominal organs. Secondly, the magnitude of the target signal seen from the isatin probes is moderate. Strategies will thus need to be developed to amplify the signal generated by the isatin probes and reduce their background uptake. In addition a very low specific activity formulation will need to be used to give the agent at a theranostic dose. Despite these challenges, the initial experience with these agents demonstrates the feasibility of generating theranostic approaches to image/modulate caspase-3 activity in vivo.

\section{Conclusion}

Molecular imaging of cell death continues to gain momentum. While the imaging of the intracellular kinases and proteases involved in cell death is in its infancy, the preclinical feasibility and utility of this approach has been well demonstrated. Future work must now focus on the development of translatable platforms to image intracellular kinase/protease activity in vivo. In addition to new diagnostic constructs, novel theranostic constructs will also need to be developed. While this is a formidable challenge it is also one that promises large rewards and is well worth pursuing.

\section{Abbreviations}

PI3K: Phosphatidylinositol 3-kinase; AKT: Akt family serine/threonine protein kinase; Gd-TO: Gadolinium-Thiazole Orange chelate; HER2: Human Epidermal Growth Factor Receptor 2; IGF: Insulin-like growth factor; EGFR: Epidermal Growth Factor Receptor; PTEN: Phosphatase and tensin homolog; PIP2: phosphatidylinositol-bisphosphate; PIP3: phosphatidylinositol-triphosphate; PDK-1: Phosphoinositide Dependent Kinase 1; BAD: Bcl-2-associated death promoter; mTOR: Mammalian Target of Rapamycin; Wm: Wortmannin; NBD: Nitro-benzoxadiazole.

\section{Acknowledgements}

This work was funded in part by the following NIH grants: R01HL093038.

\section{Conflict of Interest}

The authors have declared that no conflict of interest exists.

\section{References}

1. Hofstra L, Liem IH, Dumont EA, Boersma HH, van Heerde WL, Doevendans PA, et al. Visualisation of cell death in vivo in patients with acute myocardial infarction. Lancet. 2000; 356: 209-12.

2. Narula J, Acio ER, Narula N, Samuels LE, Fyfe B, Wood D, et al. Annexin- $\mathrm{V}$ imaging for noninvasive detection of cardiac allograft rejection. Nat Med. 2001; 7: 1347-52.

3. Sosnovik DE, Schellenberger EA, Nahrendorf M, Novikov MS, Matsui T, Dai G, et al. Magnetic resonance imaging of cardiomyocyte apoptosis with a novel magneto-optical nanoparticle. Magn Reson Med. 2005; 54: 718-24.

4. Zhao M, Beauregard DA, Loizou L, Davletov B, Brindle KM. Non-invasive detection of apoptosis using magnetic resonance imaging and a targeted contrast agent. Nat Med. 2001; 7: 1241-4.

5. Sosnovik DE, Garanger E, Aikawa E, Nahrendorf M, Figuiredo JL, Dai G, et al. Molecular MRI of cardiomyocyte apoptosis with simultaneous delayed-enhancement MRI distinguishes apoptotic and necrotic myocytes in vivo: potential for midmyocardial salvage in acute ischemia. Circ Cardiovasc Imaging. 2009; 2: 460-7.

6. Garanger E, Hilderbrand SA, Blois JT, Sosnovik DE, Weissleder $\mathrm{R}$, Josephson L. A DNA-binding Gd chelate for the detection of cell death by MRI. Chem Commun (Camb). 2009;: 4444-6.

7. Huang S, Chen HH, Yuan H, Dai G, Schuhle DT, Mekkaoui C, et al. Molecular MRI of acute necrosis with a novel DNA-binding gadolinium chelate: kinetics of cell death and clearance in infarcted myocardium. Circ Cardiovasc Imaging. 2011; 4: 729-37.

8. Hotchkiss RS, Strasser A, McDunn JE, Swanson PE. Cell death. N Engl J Med. 2009; 361: 1570-83.

9. Cantley LC. The phosphoinositide 3-kinase pathway. Science. 2002; 296: 1655-7.

10. Markman B, Dienstmann R, Tabernero J. Targeting the PI3K/Akt/mTOR pathway--beyond rapalogs. Oncotarget. 2010; 1: 530-43.

11. Manning BD, Cantley LC. AKT/PKB signaling: navigating downstream. Cell. 2007; 129: 1261-74.

12. Matsui T, Nagoshi T, Rosenzweig A. Akt and PI 3-kinase signaling in cardiomyocyte hypertrophy and survival. Cell Cycle. 2003; 2 : 220-3.

13. Communal C, Sumandea M, de Tombe P, Narula J, Solaro RJ, Hajjar RJ. Functional consequences of caspase activation in cardiac myocytes. Proceedings of the National Academy of Sciences of the United States of America. 2002; 99: 6252-6.

14. Li Q, Li B, Wang X, Leri A, Jana KP, Liu Y, et al. Overexpression of insulin-like growth factor-1 in mice protects from myocyte death after infarction, attenuating ventricular dilation, wall stress, and cardiac hypertrophy. J Clin Invest. 1997; 100: 1991-9.

15. Cortez-Retamozo V, Swirski FK, Waterman P, Yuan $\mathrm{H}$, Figueiredo JL, Newton AP, et al. Real-time assessment of inflammation and treatment response in a mouse model of allergic airway inflammation. J Clin Invest. 2008; 118: 4058-66.

16. Stangenberg L, Ellson C, Cortez-Retamozo V, Ortiz-Lopez A, Yuan H, Blois J, et al. Abrogation of antibody-induced arthritis in mice by a self-activating viridin prodrug and association with impaired neutrophil and endothelial cell function. Arthritis Rheum. 2009; 60: 2314-24.

17. Hayakawa Y, Chandra M, Miao W, Shirani J, Brown JH, Dorn GW, 2nd, et al. Inhibition of cardiac myocyte apoptosis improves cardiac function and abolishes mortality in the peripartum cardiomyopathy of Galpha(q) transgenic mice. Circulation. 2003; 108: 3036-41.

18. Holly TA, Drincic A, Byun Y, Nakamura S, Harris K, Klocke FJ, et al. Caspase inhibition reduces myocyte cell death induced by 
myocardial ischemia and reperfusion in vivo. J Mol Cell Cardiol. 1999; 31: 1709-15.

19. Yaoita H, Ogawa K, Maehara K, Maruyama Y. Attenuation of ischemia/reperfusion injury in rats by a caspase inhibitor. Circulation. 1998; 97: 276-81.

20. Matsui T, Tao J, del Monte F, Lee KH, Li L, Picard M, et al. Akt activation preserves cardiac function and prevents injury after transient cardiac ischemia in vivo. Circulation. 2001; 104: 330-5.

21. Nagoshi T, Matsui T, Aoyama T, Leri A, Anversa P, Li L, et al. PI3K rescues the detrimental effects of chronic Akt activation in the heart during ischemia/reperfusion injury. J Clin Invest. 2005; 115: 2128-38.

22. Wymann MP, Bulgarelli-Leva G, Zvelebil MJ, Pirola L, Vanhaesebroeck B, Waterfield MD, et al. Wortmannin inactivates phosphoinositide 3-kinase by covalent modification of Lys-802, a residue involved in the phosphate transfer reaction. Mol Cell Biol. 1996; 16: 1722-33.

23. Barnes KR, Blois J, Smith A, Yuan H, Reynolds F, Weissleder R, et al. Fate of a bioactive fluorescent wortmannin derivative in cells. Bioconjug Chem. 2008; 19: 130-7.

24. Blois J, Yuan H, Smith A, Pacold ME, Weissleder R, Cantley LC, et al. Slow self-activation enhances the potency of viridin prodrugs. J Med Chem. 2008; 51: 4699-707.

25. Smith A, Blois J, Yuan H, Aikawa E, Ellson C, Figueiredo JL, et al. The antiproliferative cytostatic effects of a self-activating viridin prodrug. Mol Cancer Ther. 2009; 8: 1666-75.

26. Zhang L, Lee KC, Bhojani MS, Khan AP, Shilman A, Holland EC, et al. Molecular imaging of Akt kinase activity. Nat Med. 2007; 13: 1114-9.

27. Zhang L, Bhojani MS, Ross BD, Rehemtulla A. Molecular imaging of protein kinases. Cell Cycle. 2008; 7: 314-7.

28. Zhang L, Bhojani MS, Ross BD, Rehemtulla A. Enhancing Akt imaging through targeted reporter expression. Mol Imaging. 2008; 7: 168-74.

29. Khan AP, Contessa JN, Nyati MK, Ross BD, Rehemtulla A. Molecular imaging of epidermal growth factor receptor kinase activity. Anal Biochem. 2011; 417: 57-64.

30. Nyati S, Schinske K, Ray D, Nyati M, Ross BD, Rehemtulla A. Molecular Imaging of TGFbeta-Induced Smad2/3 Phosphorylation Reveals a Role for Receptor Tyrosine Kinases in Modulating TGFbeta Signaling. Clin Cancer Res. 2011; 17: 7424-39.

31. Zhang L, Virani S, Zhang Y, Bhojani MS, Burgess TL, Coxon A, et al. Molecular imaging of c-Met tyrosine kinase activity. Anal Biochem. 2011; 412: 1-8.

32. Bhojani MS, Nyati MK, Zhao L, Normolle DP, Ross BD, Lawrence TS, et al. Molecular imaging of akt enables early prediction of response to molecular targeted therapy. Transl Oncol. 2011; 4: 122-5.

33. Niu G, Chen X. Apoptosis imaging: beyond annexin V. J Nucl Med. 2010; 51: 1659-62.

34. Maxwell D, Chang Q, Zhang X, Barnett EM, Piwnica-Worms D. An improved cell-penetrating, caspase-activatable, near-infrared fluorescent peptide for apoptosis imaging. Bioconjug Chem. 2009; 20: 702-9.

35. Ray P, De A, Patel M, Gambhir SS. Monitoring caspase-3 activation with a multimodality imaging sensor in living subjects. Clin Cancer Res. 2008; 14: 5801-9.

36. Zhang F, Zhu L, Liu G, Hida N, Lu G, Eden HS, et al. Multimodality imaging of tumor response to doxil. Theranostics. 2011; 1: 302-9.

37. Barnett EM, Zhang X, Maxwell D, Chang Q, Piwnica-Worms D. Single-cell imaging of retinal ganglion cell apoptosis with a cell-penetrating, activatable peptide probe in an in vivo glaucoma model. Proc Natl Acad Sci U S A. 2009; 106: 9391-6.

38. Chen DL, Zhou D, Chu W, Herrbrich PE, Jones LA, Rothfuss $\mathrm{JM}$, et al. Comparison of radiolabeled isatin analogs for imaging apoptosis with positron emission tomography. Nucl Med Biol. 2009; 36: 651-8.

39. Nguyen QD, Smith G, Glaser M, Perumal M, Arstad E, Aboagye EO. Positron emission tomography imaging of drug-induced tumor apoptosis with a caspase-3/7 specific [18F]-labeled isatin sulfonamide. Proc Natl Acad Sci U S A. 2009; 106: 16375-80.

40. Podichetty AK, Wagner S, Schroer S, Faust A, Schafers M, Schober $\mathrm{O}$, et al. Fluorinated isatin derivatives. Part 2. New $\mathrm{N}$-substituted 5-pyrrolidinylsulfonyl isatins as potential tools for molecular imaging of caspases in apoptosis. J Med Chem. 2009; 52: 3484-95. 